\title{
Identification of residual tumor with intraoperative contrast-enhanced ultrasound during glioblastoma resection
}

\author{
*Francesco Prada, MD, ${ }^{1}$ Massimiliano Del Bene, MD, ${ }^{1}$ Riccardo Fornaro, MD, ${ }^{1}$ \\ Ignazio G. Vetrano, MD, ${ }^{1}$ Alberto Martegani, MD, ${ }^{2}$ Luca Aiani, MD, ${ }^{2}$ \\ Luca Maria Sconfienza, MD, PhD, ${ }^{3}$ Giovanni Mauri, MD, ${ }^{3}$ Luigi Solbiati, MD, ${ }^{4}$ Bianca Pollo, MD, ${ }^{5}$ \\ and Francesco DiMeco, MD ${ }^{1,6}$
}

\begin{abstract}
Departments of ${ }^{1}$ Neurosurgery and ${ }^{5}$ Neuropathology, Fondazione IRCCS Istituto Neurologico C. Besta, Milan; ${ }^{3}$ Department of Radiology, IRCCS Policlinico San Donato, Milan; ${ }^{2}$ Department of Radiology, Ospedale Valduce, Como; ${ }^{4}$ Department of Radiology, Humanitas Research Hospital, Rozzano, Italy; and ${ }^{6}$ Department of Neurosurgery, Johns Hopkins Medical School, Baltimore, Maryland
\end{abstract}

OBJECTIVE The purpose of this study was to assess the capability of contrast-enhanced ultrasound (CEUS) to identify residual tumor mass during glioblastoma multiforme (GBM) surgery, to increase the extent of resection.

METHODS The authors prospectively evaluated 10 patients who underwent surgery for GBM removal with navigated ultrasound guidance. Navigated B-mode and CEUS were performed prior to resection, during resection, and after complete tumor resection. Areas suspected for residual tumors on B-mode and CEUS studies were localized within the surgical field with navigated ultrasound and samples were sent separately for histopathological analysis to confirm tumor presence.

RESULTS In all cases tumor remnants were visualized as hyperechoic areas on B-mode, highlighted as CEUS-positive areas, and confirmed as tumoral areas on histopathological analysis. In 1 case only, CEUS partially failed to demonstrate residual tumor because the residual hyperechoic area was devascularized prior to ultrasound contrast agent injection. In all cases CEUS enhanced B-mode findings.

CONCLUSIONS As has already been shown in other neoplastic lesions in other organs, CEUS is extremely specific in the identification of residual tumor. The ability of CEUS to distinguish between tumor and artifacts or normal brain on $\mathrm{B}$-mode is based on its capacity to show the vascularization degree and not the echogenicity of the tissues. Therefore, CEUS can play a decisive role in the process of maximizing GBM resection.

http://thejns.org/doi/abs/10.3171/2015.11.FOCUS15573

KEY WORDS intraoperative imaging; contrast-enhanced ultrasound; intraoperative ultrasound; extent of resection; residual tumor; glioblastoma

$\mathrm{G}$ LIOBLASTOMA multiforme (GBM) is the most aggressive among the glial tumors. It is a WHO Grade IV astrocytoma and unfortunately it is also the most frequent, representing $15.6 \%$ of all primitive nervous system tumors and $45.2 \%$ of the malignant ones. ${ }^{19}$ Given its poor prognosis and its incidence, GBM treatment is the object of numerous investigations. Despite the intense effort to find a more effective therapy, currently the standard treatments guarantee a median overall survival of only $12-15$ months. ${ }^{35,36}$ The mainstay of treatment for newly diagnosed GBM is resection followed by radiation therapy and chemotherapy. A crucial prognostic factor in oncological neurosurgery is the extent of resection (EOR). Several studies have addressed the importance of EOR in GBM surgery. ${ }^{4,9,17,28}$

All of these findings have highlighted the need to obtain

ABBREVIATIONS CEUS = contrast-enhanced ultrasound; EOR = extent of resection; GBM = glioblastoma multiforme; iCT, iMRI, iUS = intraoperative CT, MRI, US; UCA = ultrasound contrast agent; 5-ALA = 5-aminolevulinic acid.

SUBMITTED November 1, 2015. ACCEPTED November 30, 2015

INCLUDE WHEN CITING DOI: 10.3171/2015.11.FOCUS15573.

* Drs. Prada and Del Bene contributed equally to this work. 
a subtotal or a gross-total resection as first-line therapy for GBM; indeed, the smaller the residual tumor burden, the fewer the number of tumor cells that need to be targeted with adjuvant therapy. In this effort, tumor visualization is a key factor to maximize the EOR. Numerous solutions exist: neuronavigation, fluorescence, and intraoperative imaging (MRI, CT, ultrasound).

Neuronavigation allows the use preoperative acquired images such as CT, MRI, functional MRI, and diffusion tensor imaging to achieve orientation in the surgical field. ${ }^{2}$ Neuronavigation is extremely helpful in finding the tumor and the surrounding neurovascular structures, but it is affected by brain shift and brain deformation that progressively degrade the information during surgery. $7,16,18$

Fluorescence-guided surgery with 5-aminolevulinic acid (5-ALA) allows the identification of tumor tissue with great accuracy, but only on the surface of the surgical cavity; to categorize an area as 5-ALA positive, it is necessary to expose and evaluate it in blue light. In other words, 5-ALA does not allow a complete overview of the tumor; for example, 5-ALA cannot show a residual mass if its surface is not directly exposed. ${ }^{34}$

Intraoperative MRI and CT (iMRI and iCT) are able to counteract brain shift and brain deformation and offer high spatial resolution and a wide field of view, but they are expensive as well as time- and space-consuming. Additionally, it is not possible to operate under direct imaging guidance; consequently they cannot be considered real-time intraoperative imaging modalities. ${ }^{1,3}$

Intraoperative ultrasound (iUS) has been used in neurosurgery since the early 1980 s, and over the years a lot of applications have been reported. ${ }^{26}$ Intraoperative ultrasound is a true real-time, dynamic technique that offers a good temporal and spatial resolution..$^{1,2,6,12,14,31}$ Its high spatial resolution permits an accurate tissue differentiation, which has been shown to improve the EOR in glioma surgery. ${ }^{2}$ The main problem in iUS B-mode in glioma surgery is the challenging discrimination between residual tumor, tumor-induced edema, and surgically induced artifacts. Especially at the end of the surgery it is difficult to assess if a hyperechoic area is truly a residual tumor. ${ }^{13,29,32,33}$ Contrast-enhanced US (CEUS) is an iUS modality that uses an ultrasound contrast agent (UCA) to improve the contrast between tumor, healthy tissue, and artifacts.

In other organs, such as the liver, CEUS is routinely used as a rapid, cost-effective, and precise diagnostic tool to differentiate between malignant and benign lesions and to determine tumor localization. ${ }^{10,11}$ It has a sensitivity up to $90 \%$ and a specificity of $99 \%$, with an accuracy of $89 \%$ in the diagnosis of malignant liver lesions. ${ }^{38}$ The CEUS technique is also used intraoperatively for the detection of liver malignancies, and it has an impact on surgical strategy for both primary and metastatic tumors, increasing the correctness of B-mode findings up to $20 \%$. $^{8}$

We have already demonstrated that CEUS can highlight all glial tumors, particularly GBMs, with a specific contrast enhancement, which also permits their characterization $^{24,25}$ and visualization in the surgical volume. In this study our goal was to evaluate the role of CEUS in identifying residual tumor mass in GBM surgery, thus enhancing tumor resection.

\section{Methods}

\section{Patient Population}

We performed CEUS routinely during brain tumor removal, and also before and after tumor resection. We prospectively evaluated 10 patients harboring a cerebral GBM (WHO Grade IV) who underwent surgery for brain tumor removal, in whom we performed multiple CEUS scans. All patients were in good general status (American Society of Anesthesiologists Class I-III; Karnofsky Performance Scale score > 70). All patients were systematically informed about the surgical procedure, and written consent was obtained. During the completion of the present study we never changed the usual or standard treatment of these patients, and the use of the UCA was approved by our institutional review board.

\section{Study Equipment}

We used the newest-generation iUS system equipped with an electromagnetic tracking system allowing neuronavigation (Esaote MyLab with Virtual Navigator). Neuronavigation is based on fusion imaging between iUS and the corresponding preoperative volumetric T1-weighted Gd-enhanced MRI slice, displayed in a coplanar fashion (Fig. 1). This system allows practitioners to navigate both the ultrasound probe and the navigation pointer in an electromagnetic field, using multiple tracked devices. As our ultrasound probe we used a linear-array multifrequency (3-11 MHz) device. During the surgical procedure the probe was covered in a sterile plastic sheet, with sterile ultrasound coupling gel (Civco)., ${ }^{1,21-25}$

As a UCA we used sulfur hexafluoride-filled lipidic microbubbles, a second-generation UCA (SonoVue), which was injected in a peripheral vein as a bolus $(2.4 \mathrm{ml}[5 \mathrm{mg} /$ $\mathrm{ml}]$ ). The CEUS technique was performed using a specific algorithm (contrast-tuned imaging) that decreases the ultrasound mechanical index value needed to obtain microbubble resonance for harmonic imaging, and represents only the specific echo signal from the microbubble resonance. ${ }^{20,23-25,30}$

\section{Study Procedure}

After bone flap removal but before dura mater opening, we acquired a navigated iUS B-mode scan to identify the lesion along with surrounding healthy parenchyma, to evaluate the accuracy of fusion imaging registration, and to create a preresection tumor volume (Fig. 2). We then performed an initial navigated CEUS examination to recognize arterial feeders, degree of vascularization, and venous drainage, and to assess lesion contrast enhancement, comparing it to the corresponding B-mode and preoperative MR image, thus creating a multimodal preresection tumor volume. The CEUS technique was performed according to the European Federation of Societies for Ultrasound in Medicine and Biology (EFSUMB) guidelines. ${ }^{20,30}$

During surgery we performed multiple standard Bmode scans. Compensation for brain shift was accomplished using the fine-tuning option as previously described in other papers from our group, ${ }^{21,22}$ to maintain the surgical volume.

Our protocol comprises 3 navigated B-mode and CEUS 

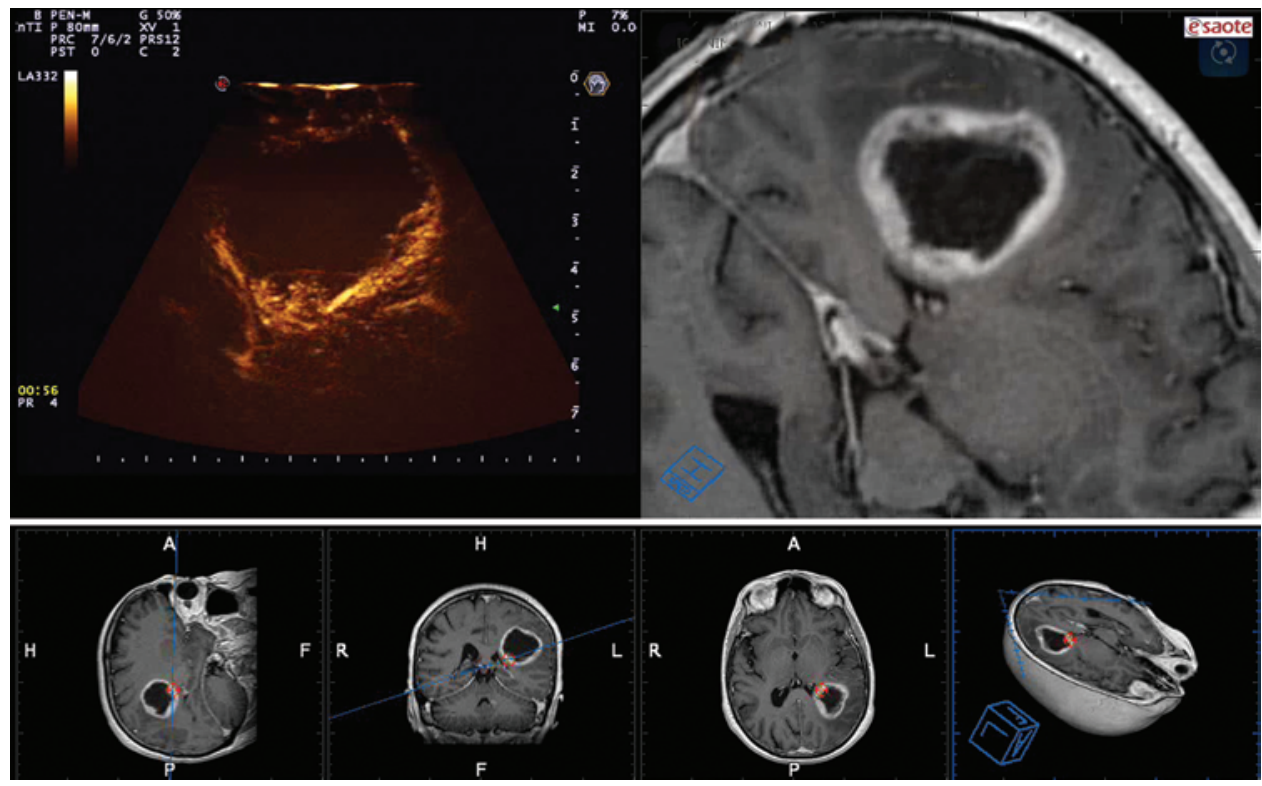

FIG. 1. Navigated CEUS screenshot in a case of left parietal GBM. Upper: The CEUS image is depicted on the left side of the panel, together with the corresponding coplanar preoperative MRI study on the right. Lower: In this panel, 4 reconstructions of preoperative MR images are presented.

acquisitions prior to, during, and after tumor removal, to evaluate the surgical cavity (Fig. 3). When a hyperechoic area within the cavity was noted on B-mode, navigated CEUS was performed. The enhanced area was then localized within the previously defined surgical volume and resected under direct CEUS guidance, for which the navi- gated pointer was used-the pointer was visible both on real-time ultrasound and preoperative MRI. The lesion's appearance and localization on B-mode, CEUS, and navigation was recorded. The CEUS-positive areas were removed and sent separately from the main tumor specimen for histopathological analysis to confirm the presence of
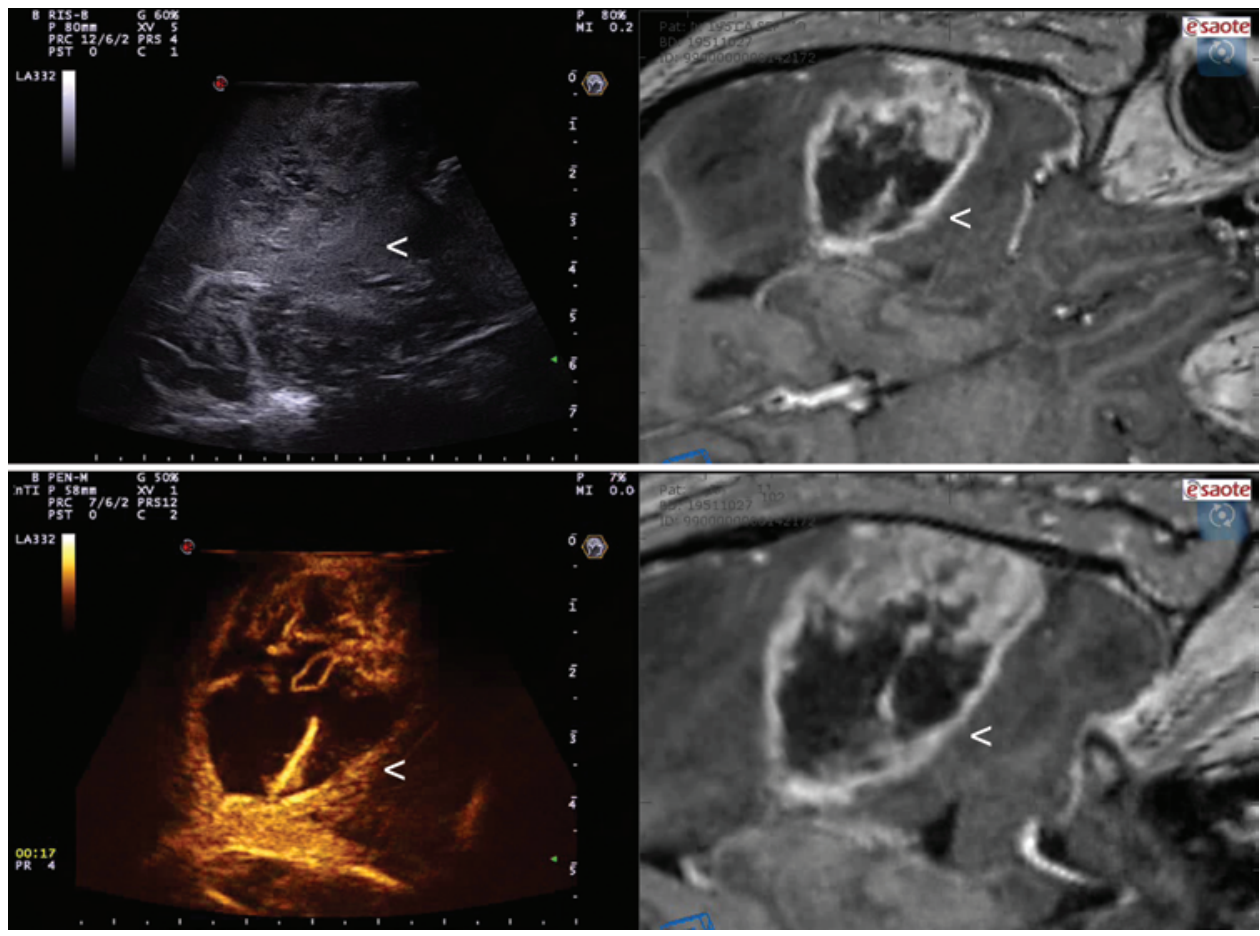

FIG. 2. Preresection transdural B-mode and CEUS scans in a case of left temporal GBM. The B-mode (upper panel, left) and CEUS (lower panel, left) images are presented together with the corresponding coplanar preoperative MRI sequences (upper and lower panels, right). In B-mode it is difficult to discern between tumor and tumor-induced edema (arrowheads), whereas with CEUS the tumor borders are highlighted and superimposable onto preoperative MR images (arrowheads). 

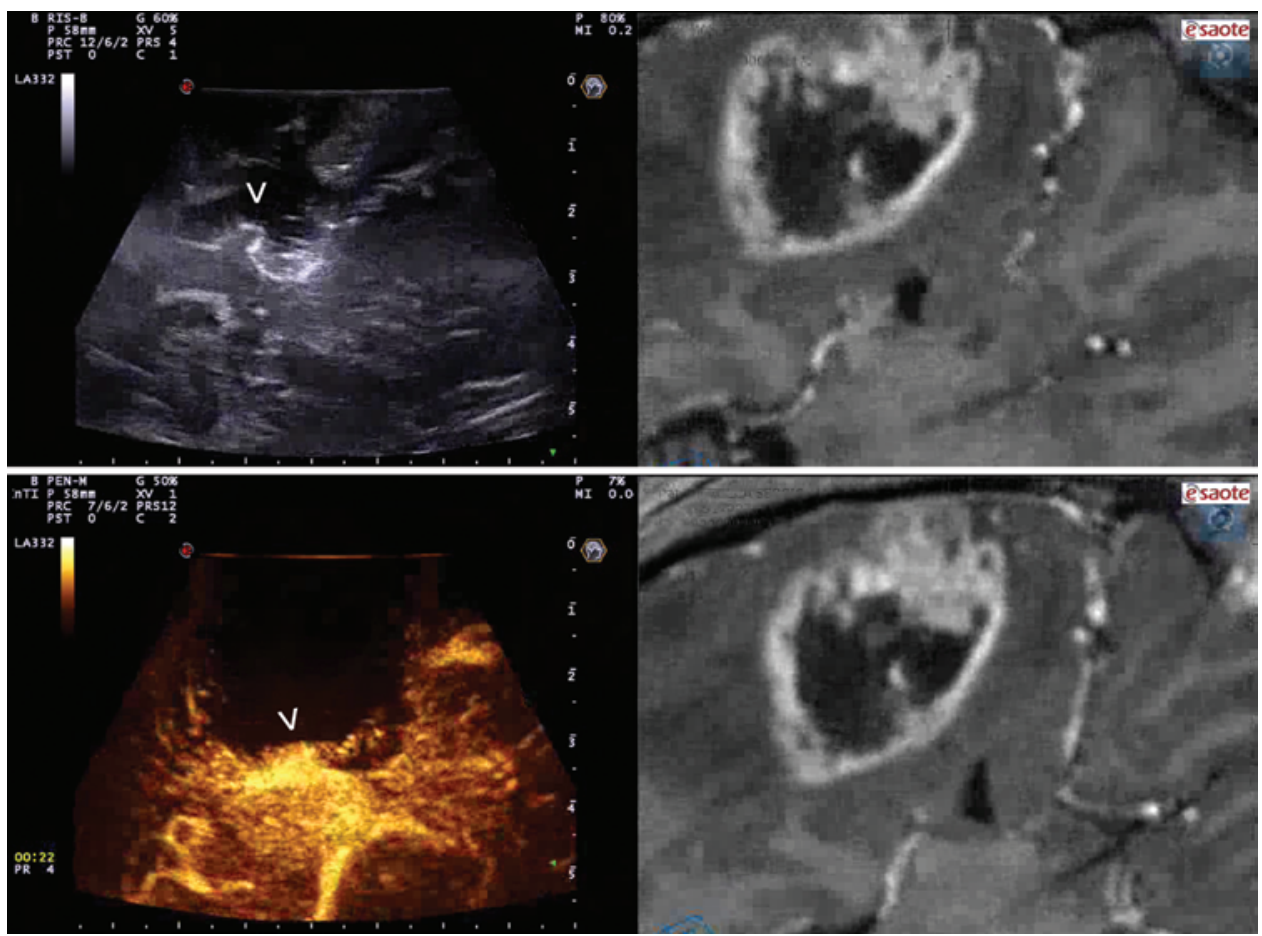

FIG. 3. The B-mode and CEUS scans after subtotal resection in a case of left temporal GBM. The B-mode (upper panel, left) and CEUS (lower panel, left) images are presented together with the corresponding coplanar preoperative MRI sequences (upper and lower panels, right). In B-mode some hyperechoic artifacts appeared (arrowheads), making it difficult to discern between tumor remnants and edematous brain tissue, whereas in CEUS the area underlying the artifacts is clearly identifiable as an enhanced component of the tumor located within the navigated surgical volume (arrowheads).

the tumor. Each CEUS-positive area was also compared with B-mode findings (hyperechoic or hypoechoic) on preoperative MRI (Table 1).

Data analysis was based on a qualitative comparison between B-mode and CEUS, using histopathological findings to confirm if hyperechoic areas on B-mode with enhancement after UCA administration corresponded to tumor remnants, therefore assessing the accuracy of CEUS in highlighting residual tumor.

\section{Results}

We evaluated 10 patients (5 men and 5 women), who had a mean age of 63.8 years and a mean Karnofsky Performance Scale score of 80 (range 50-90). All patients undergoing surgery had been newly diagnosed with brain tumor that had features suggestive for GBM (WHO IV) on preoperative imaging; all cases considered for the analysis were confirmed by a pathologist to be GBM. We did not observe any adverse event or side effect related to iUS scans or to the administration of the UCA.

In all 10 cases we were able to visualize the lesion on B-mode and CEUS prior to resection. After subtotal tumor removal, hyperechoic areas were noted on B-mode within the surgical cavity and were afterward highlighted with CEUS. Using navigated ultrasound, the highlighted areas were further resected and confirmed to be tumor remnants on histopathological analysis in all cases.

In all cases we were able to visualize the lesion along with surrounding brain parenchyma with B-mode, before opening the dura mater (Fig. 1). General features of a GBM in B-mode are the hyperechogenicity to brain parenchyma, with a homo- or heterogeneous appearance composed of multiple well-defined nodular or cystic areas and circumscribed or diffuse margins (Table 1).

The main problem with B-mode was related to the presence of artifacts due to surgical maneuvers, which we found in different degrees in all cases. During tumor removal, the B-mode findings became difficult to interpret in all cases because of the presence of hyperechoic areas surrounding the surgical cavity that could have been interpreted as residual tumor, edema, debris, blood clots, or artifacts (Figs. 3-5).

Navigation system spatial error was found to be less than $2 \mathrm{~mm}$ in all cases. This spatial error in fusion imaging between iUS images and preoperative MRI was manually corrected through fine-tuning every time it was necessary. Thanks to the possibility of correcting the brain shift, we were able to use a navigated ultrasound probe during all stages of surgery in all cases, and also in the postresection iUS evaluation of potential residual tumor (Fig. 3). The main limitation of the fine-tuning is that it can fix only brain shift and not brain deformation, and that navigation relies on preoperative imaging that does not describe the real intraoperative situation. However, the navigated ultrasound probe and pointer allowed us to correctly localize the highlighted tissue within the surgical volume in all 10 cases.

In all cases evaluated with CEUS, before opening the dura mater, we were able to visualize and to study the contrast enhancement phases of each lesion (Figs. 2 and 6). 


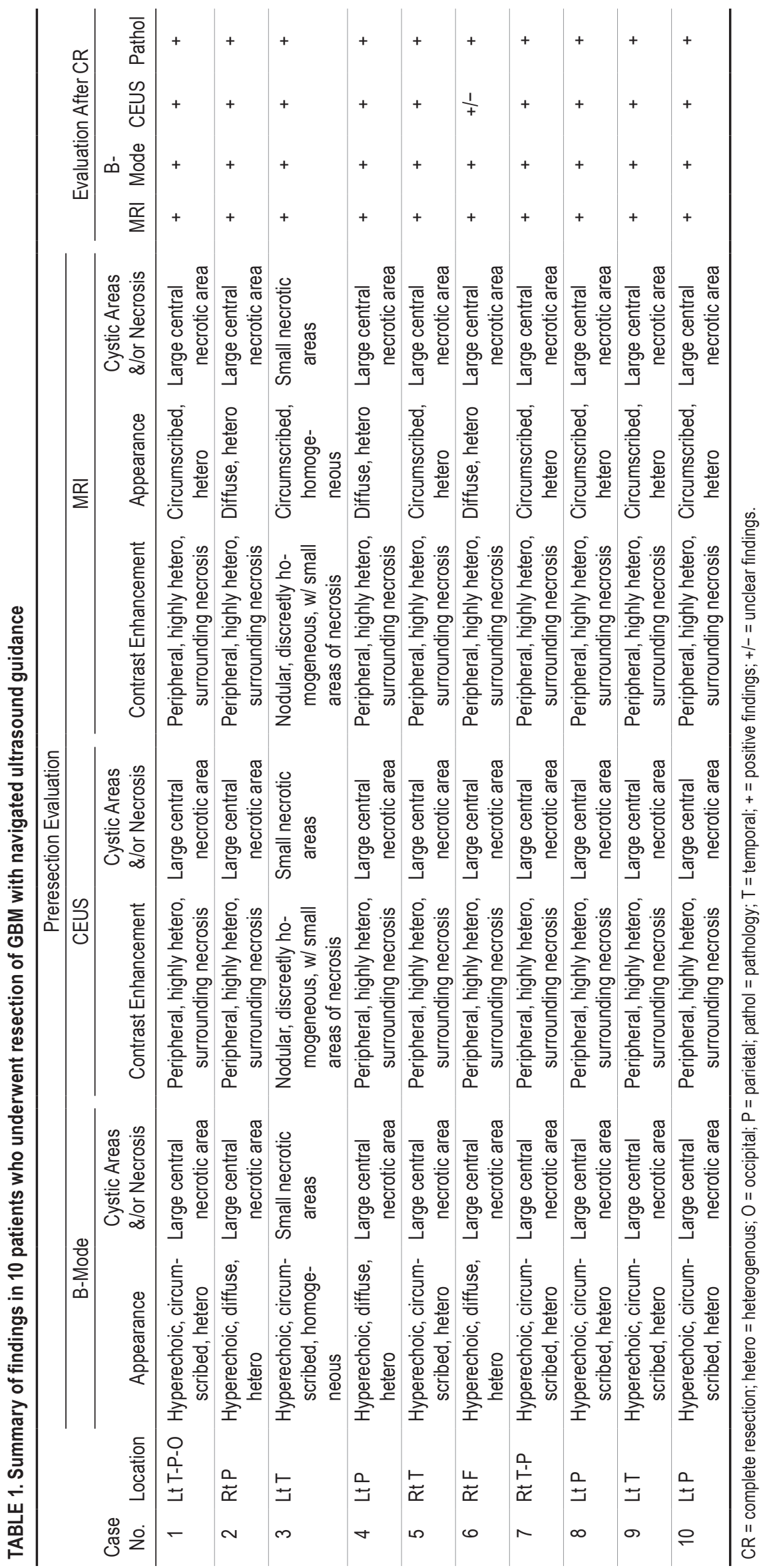



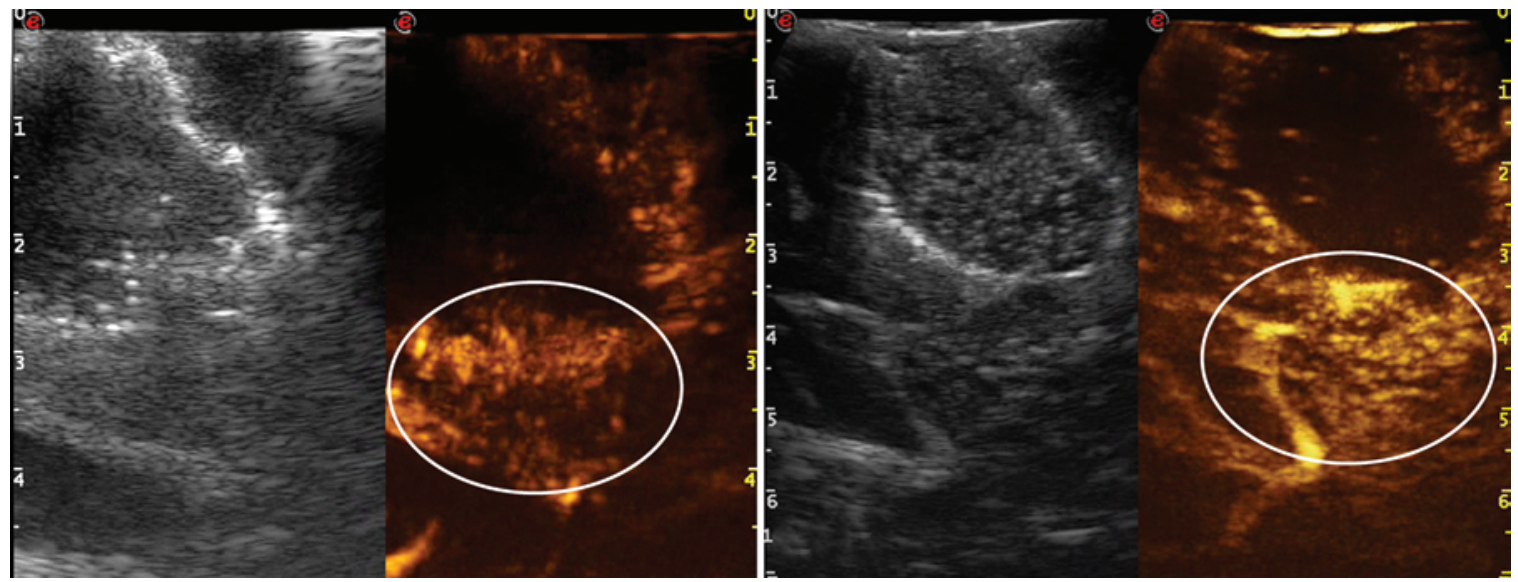

FIG. 4. Examples of residual tumor identification. Left: Dual display of low mechanical index B-mode and corresponding CEUS scans in a case of frontal right GBM. Right: Dual display of low mechanical index B-mode and corresponding CEUS scans in a case of temporoparietooccipital GBM. The artifacts on the surface of the surgical cavity might be misleading when judging the hyperechoic residual tumor mass in B-mode, whereas this is more easily identifiable in CEUS (circles).

In general, GBMs showed a contrast enhancement (20-30 seconds after UCA injection) characterized by a rapid arterial phase (2-3 seconds), followed by a rapid contrast enhancement peak (3-5 seconds). The arterial supply was clearly visible through many macrovessels within the lesion; typically the contrast enhancement progression was centripetal. The venous phase was rapid (5-10 seconds); usually the drainage system was composed of multiple veins aiming toward the periventricular zone (Fig. 6).

In the late stages of surgical removal, in 9 cases we were able to visualize areas of contrast enhancement in conti- nuity with the surgical cavity (Table 1 and Figs. 3 and 4). In only 1 case was there reduced contrast enhancement compared with B-mode, probably due to the partial devascularization of the residual tumor (Table 1 and Fig. 7). In all cases, residual tumor tissue was highlighted with CEUS after subtotal tumor resection, and was confirmed to be neoplastic on a separate analysis.

\section{Discussion}

Intraoperative CEUS performed during GBM resection
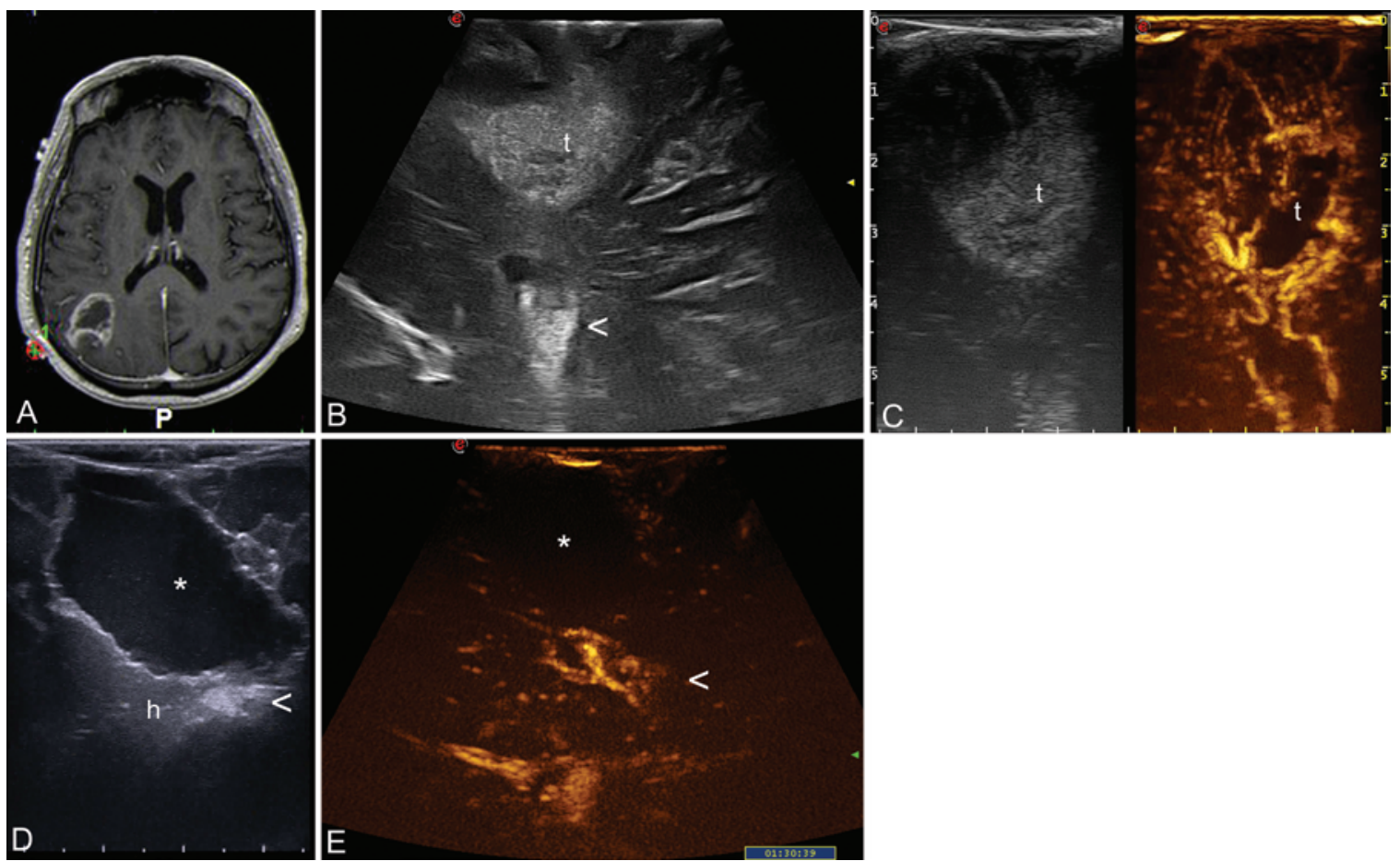

FIG. 5. Preresection (A-C) and postresection ( $D$ and $\mathbf{E})$ scans in a case of temporoparietooccipital GBM. Preoperative T1weighted MRI study obtained with Gd contrast (A). Preresection B-mode (B), and a dual display of low mechanical index B-mode and CEUS scans (C). End of resection on B-mode (D) and CEUS (E) scans. The asterisks designate the surgical cavity, and the arrowheads denote the choroidal plexus. $\mathrm{h}=$ hyperechoic artifacts; $\mathrm{t}=$ tumor. 

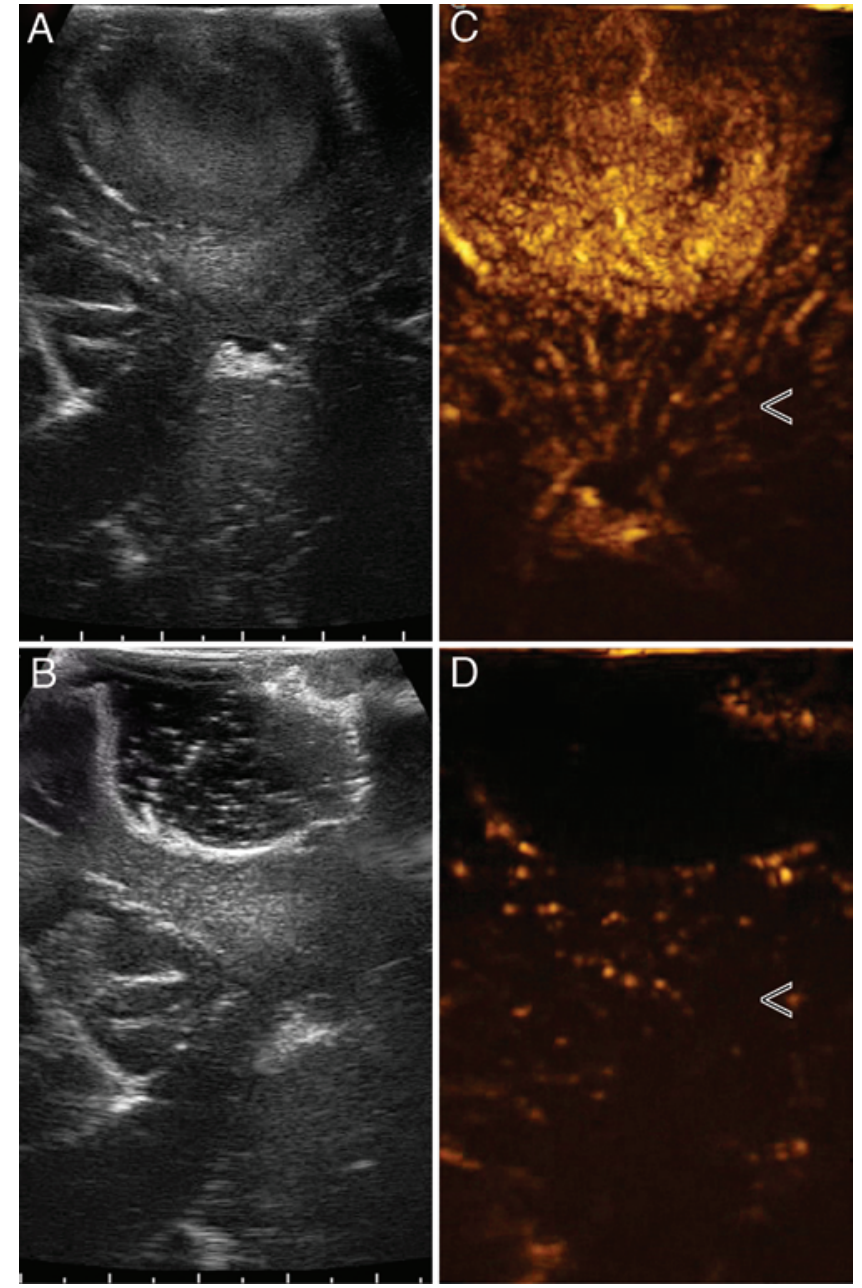

FIG. 6. Intraoperative evaluation on B-mode (A and B) and CEUS (C and D) scans of a frontal GBM. In $\mathrm{C}$, the CEUS venous phase shows multiple small draining veins toward the ventricle (arrowhead). After total tumor resection (D), the draining vessels have disappeared in CEUS (arrowhead).

allowed us to highlight the residual tumor tissue (detected as a hyperechoic area in B-mode) with great accuracy, overcoming the difficulties of B-mode interpretation caused by artifacts, edema, and surgical manipulation. In all cases the CEUS-positive area was confirmed to be neoplastic on histopathological analysis (Table 1). In only 1 case, CEUS underestimated the residual tumor, showing a lighter and slower contrast enhancement compared with the preresection CEUS, in an area that was within the tumor (Fig. 7). This was probably due to the partial devascularization of the residual tumor; with a reduced tissue perfusion the distribution of the intravascular UCA was impaired.

The B-mode evaluation after subtotal tumor resection was not helpful in classifying a hyperechoic area as residual tumor, mainly because of artifacts and surgical manipulation $^{13,27,29,32,33}$ (Figs. 3-5). Even B-mode comparison with preoperative MRI through fusion imaging was not completely reliable because of the inability to take into account brain deformation, mainly toward the surface. ${ }^{6,7,16,18}$

Artifacts are in fact the main limitation in iUS B-mode
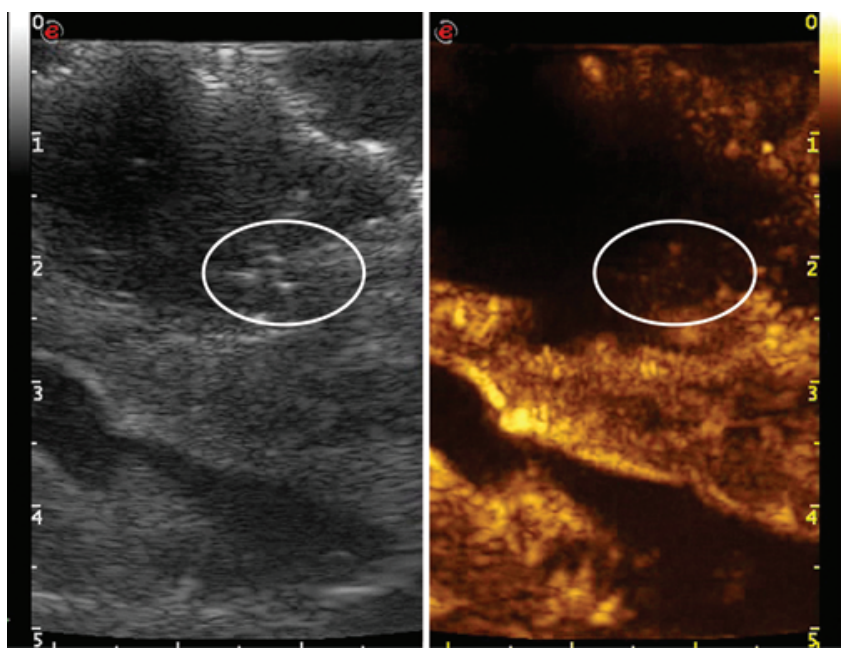

FIG. 7. Dual-mode scan performed after subtotal resection. The CEUS scan (right) demonstrates only mild contrast enhancement, but a residual tumor is present and visible as a hyperechoic area on B-mode (left) (circles). This area was resected and histological analysis confirmed that it was residual tumor. This was probably due to the fact that the CEUSnegative area had been devascularized prior to imaging, leading to an impaired distribution of the UCA in the residue.

interpretation in glioma surgery. ${ }^{13,27,29,32,33}$ Surgical maneuvers inevitably lead to difficulty in ultrasound discrimination between residual tumor and surgically induced edema or artifacts. In particular, at the end of tumor resection it is difficult to assess if a hyperechoic area is actually residual tumor (Figs. 3-5). Another problem is related to the transit of the ultrasound beam through media having different attenuation coefficients and to the presence of blood or hemostatic materials on the walls of the surgical cavity. Indeed, both of these factors lead to hyperechogenicity of the border and to attenuation of the ultrasound beam below the surgical bed. Moreover, because the surgical bed is not homogeneous but extremely indented, the ultrasound beam is subjected to reflection and transmission in a changeable manner. . $^{13,27,29,32,33}$

To overcome these problems and to improve image quality, several solutions have been introduced. Thanks to the possibility of navigating the iUS probe, it is possible to compare the location of a hyperechoic area on iUS with the location of the tumor on preoperative MRI, when the studies are displayed in a coplanar fashion. If the hyperechoic area is located outside tumor boundaries prior to resection, it is most likely to be an artifact. This finding is possible using a navigated iUS device. 5,15,21-23,27,32 Anyway, this solution has major limitations because of the impossibility of taking into account not brain deformation but only brain shift. For this reason the comparison between iUS and preoperative MRI can only be suggestive.

Another approach to reduce artifacts was proposed by Selbekk et al. in 2013 and consists of the use of a fluid with specific acoustic properties that mimics brain tissue. ${ }^{29}$ The rationale behind the use of this fluid is that the ultrasound beam is transmitted through a medium that has the same attenuation capacity as the underlying brain parenchyma, avoiding the enhanced brightness below the surgical bed. The proposed fluid has the same attenuation coefficient as 
the human brain and is sufficiently viscous to be removed with suction after iUS imaging. However, this solution is still under development and is far from being routinely used in a clinical setting; moreover, it can only reduce the bright areas below the cavity.

Steňo and colleagues found a source of brightness artifacts in the distance between the probe and the bottom of the cavity, because the ultrasound beam has to penetrate a higher column of water. ${ }^{32,33}$ To overcome this limitation, these investigators propose an intracavitary scan with a miniature high-frequency probe..$^{5,32,33}$ In our opinion, this solution is not free from disadvantages; indeed, when using a small probe in the depth of a surgical cavity it is not possible to obtain an overview of anatomy, residual mass entity, and extension because of the limited field of view of the probe, limited lateral resolution, and limited ultrasound penetration. . $^{13,29}$

In this context CEUS is extremely interesting, because it can highlight tumor tissue without relying on its echogenicity but on its vascularization ${ }^{20,23-25,30}$ (Figs. 3-6). Indeed CEUS is a harmonic imaging modality that depicts the distribution of microbubbles in a tissue. Thanks to their structure, sulfur hexafluoride-filled lipidic microbubbles cannot diffuse to the interstitial space, giving a representation of only the vascular district. The degree of contrast enhancement is a consequence of the density of the capillaries, which in turn is proportional to tissue activity. ${ }^{20,23-25,30}$

Furthermore, CEUS is independent from angle of insonation, it is repeatable, and if compared with Doppler imaging, it can show simultaneously both high- and lowflow vessels without the need of a specific setting. The CEUS technique has a very good spatial and temporal resolution, and thanks to the harmonic imaging feature it is extremely specific..$^{20,23-25,30}$ The CEUS technique is used in several organs both in diagnostic and intraoperative settings, because it allows practitioners to differentiate between benign and malignant lesions and helps in localizing the target and controlling treatment efficacies. ${ }^{37,38}$

In glioma surgery, CEUS is capable of highlighting the lesions and defining their borders compared with standard B-mode. It allows the identification of the more viable areas for biopsies and the characterization of different grades of glioma. ${ }^{24}$ The UCA used in this study is a safe drug with minor contraindications and can be repeated multiple times during surgery..$^{20,23-25,30}$ We did not experience any adverse event in our series.

In the specific case of GBMs, CEUS gave an important contrast enhancement in proliferating areas and, on the contrary, no contrast enhancement at all in necrotic zones and surrounding brain parenchyma ${ }^{24,25}$ (Figs. 1, 2, and 5). Therefore, we deemed CEUS to be an important tool also in tumor remnant detection. When residual tumor tissue is present after subtotal tumor removal, CEUS can demonstrate its presence, unless the observed area has not been previously devascularized during partial removal (Figs. 3, 4 , and 7).

In our series CEUS highlighted the lesion and showed tumor remnants in all cases, even though in 1 case the UCA circulation was slightly impaired after resection. Therefore, the ability of CEUS to show neoplastic tissue in GBM surgery prior to, during, and after tumor resection might become a pivotal tool for tumor identification and might enhance tumor removal, particularly if coupled with other complementary intraoperative techniques such as fluorescence imaging. In fact CEUS shows a tomographic section of the surgical field, whereas fluorescence imaging allows direct tumor visualization on its surface. Their synergistic application and data integration might be helpful in further enhancing real-time tumor visualization and resection.

Indeed, CEUS has some drawbacks. A contrast-specific algorithm, present only in high-end ultrasound equipment, is mandatory to obtain the required harmonic imaging. Also, CEUS is an operator-dependent technique, and therefore specific training is necessary to regulate the settings, such as mechanical index and ultrasound focus, and also for image interpretation, which is not straightforward.

The UCA preparation also has to be accurate, because it might sometimes lead to ineffective CEUS imaging. It should also be kept in mind that UCAs are purely intravascular agents and that tumor remnant visualization with CEUS should be performed prior to coagulation of tumor feeding vessels and resection, to allow UCA to reach the area of observation; otherwise visualization of the residual tumor will be impaired.

Nevertheless, when properly managed, CEUS allows for real-time visualization of both micro- and macrocirculation, of small and large vessels, and of low- and highflow vessels, regardless of the angle of insonation. ${ }^{20,23-25,30}$ However, all of these limitations can be addressed with adequate training and experience. In our series we were able to detect GBMs intraoperatively with CEUS in all cases. We report qualitative observational data in this work; our findings have to be confirmed by larger series that also include biopsies, not only in B-mode- or CEUS-positive areas within the preoperative tumor volume but also outside, to calculate positive and negative predictive values. We also did not calculate the influence of the CEUS on the EOR, nor we did we correlate it with MRI findings, because this was not the aim of our study. Indeed, further studies are needed to compare CEUS findings with those of other intraoperative imaging techniques.

\section{Conclusions}

Our results demonstrate that iUS and particularly CEUS have an effective and specific role in identifying residual tumor in GBM surgery. Therefore, UCAs can play a decisive role in the process of maximizing resection because they precisely identify and locate neoplastic tissue, both prior to and after resection, allowing for safe removal. The CEUS modality is a true real-time, repeatable, readily available, and relatively inexpensive technique that allows practitioners to address major standard B-mode drawbacks. Its synergistic use with other imaging modalities could indeed improve GBM visualization and resection.

\section{Acknowledgments}

The research leading to these results has received funding from the European Union Seventh Framework Programme (FP7/20072013) under grant agreement no. 602 923. We thank Mrs. Caroline King, BArch, for her help in revising the manuscript. 


\section{References}

1. Almeida JP, Chaichana KL, Rincon-Torroella J, Quinones-Hinojosa A: The value of extent of resection of glioblastomas: clinical evidence and current approach. Curr Neurol Neurosci Rep 15:517, 2015

2. Barone DG, Lawrie TA, Hart MG: Image guided surgery for the resection of brain tumours. Cochrane Database Syst Rev 1:CD009685, 2014

3. Black PM, Moriarty T, Alexander E III, Stieg P, Woodard EJ, Gleason PL, et al: Development and implementation of intraoperative magnetic resonance imaging and its neurosurgical applications. Neurosurgery 41:831-845, 1997

4. Chaichana KL, Jusue-Torres I, Navarro-Ramirez R, Raza SM, Pascual-Gallego M, Ibrahim A, et al: Establishing percent resection and residual volume thresholds affecting survival and recurrence for patients with newly diagnosed intracranial glioblastoma. Neuro Oncol 16:113-122, 2014

5. Coburger J, König RW, Scheuerle A, Engelke J, Hlavac M, Thal DR, et al: Navigated high frequency ultrasound: description of technique and clinical comparison with conventional intracranial ultrasound. World Neurosurg 82:366-375, 2014

6. Dohrmann GJ, Rubin JM: History of intraoperative ultrasound in neurosurgery. Neurosurg Clin N Am 12:155166, ix, 2001

7. Dorward NL, Alberti O, Velani B, Gerritsen FA, Harkness WF, Kitchen ND, et al: Postimaging brain distortion: magnitude, correlates, and impact on neuronavigation. $\mathbf{J}$ Neurosurg 88:656-662, 1998

8. Konopke R, Bunk A, Kersting S: The role of contrastenhanced ultrasound for focal liver lesion detection: an overview. Ultrasound Med Biol 33:1515-1526, 2007

9. Lacroix M, Abi-Said D, Fourney DR, Gokaslan ZL, Shi W, DeMonte F, et al: A multivariate analysis of 416 patients with glioblastoma multiforme: prognosis, extent of resection, and survival. J Neurosurg 95:190-198, 2001

10. Martegani A, Aiani L, Borghi C: The use of contrastenhanced ultrasound in large vessels. Eur Radiol 14 (Suppl 8):73-86, 2004

11. Mauri G, Porazzi E, Cova L, Restelli U, Tondolo T, Bonfanti $\mathrm{M}$, et al: Intraprocedural contrast-enhanced ultrasound (CEUS) in liver percutaneous radiofrequency ablation: clinical impact and health technology assessment. Insights Imaging 5:209-216, 2014

12. Moiyadi A, Shetty P: Objective assessment of utility of intraoperative ultrasound in resection of central nervous system tumors: A cost-effective tool for intraoperative navigation in neurosurgery. J Neurosci Rural Pract 2:4-11, 2011

13. Moiyadi AV: Linear intraoperative ultrasound probes and phased-array probes: two sides of the same coin. Acta Neurochir (Wien) 157:957-958, 2015

14. Moiyadi AV: Objective assessment of intraoperative ultrasound in brain tumors. Acta Neurochir (Wien) 156:703-704, 2014

15. Moiyadi AV, Kannan S, Shetty P: Navigated intraoperative ultrasound for resection of gliomas: Predictive value, influence on resection and survival. Neurol India 63:727-735, 2015

16. Nimsky C, Ganslandt O, Cerny S, Hastreiter P, Greiner G, Fahlbusch R: Quantification of, visualization of, and compensation for brain shift using intraoperative magnetic resonance imaging. Neurosurgery 47:1070-1080, 2000

17. Orringer D, Lau D, Khatri S, Zamora-Berridi GJ, Zhang K, $\mathrm{Wu} \mathrm{C}$, et al: Extent of resection in patients with glioblastoma: limiting factors, perception of resectability, and effect on survival. J Neurosurg 117:851-859, 2012

18. Orringer DA, Golby A, Jolesz F: Neuronavigation in the surgical management of brain tumors: current and future trends. Expert Rev Med Devices 9:491-500, 2012
19. Ostrom QT, Gittleman H, Farah P, Ondracek A, Chen Y, Wolinsky Y, et al: CBTRUS statistical report: Primary brain and central nervous system tumors diagnosed in the United States in 2006-2010. Neuro Oncol 15 (Suppl 2):ii1-ii56, 2013

20. Piscaglia F, Nolsøe C, Dietrich CF, Cosgrove DO, Gilja $\mathrm{OH}$, Bachmann Nielsen M, et al: The EFSUMB Guidelines and Recommendations on the Clinical Practice of Contrast Enhanced Ultrasound (CEUS): update 2011 on non-hepatic applications. Ultraschall Med 33:33-59, 2012

21. Prada F, Del Bene M, Mattei L, Casali C, Filippini A, Legnani F, et al: Fusion imaging for intra-operative ultrasound-based navigation in neurosurgery. J Ultrasound 17:243-251, 2014

22. Prada F, Del Bene M, Mattei L, Lodigiani L, DeBeni S, Kolev V, et al: Preoperative magnetic resonance and intraoperative ultrasound fusion imaging for real-time neuronavigation in brain tumor surgery. Ultraschall Med 36:174-186, 2015

23. Prada F, Del Bene M, Moiraghi A, Casali C, Legnani FG, Saladino A, et al: From grey scale B-mode to elastosonography: multimodal ultrasound imaging in meningioma surgery-pictorial essay and literature review. BioMed Res Int 2015:925729, 2015

24. Prada F, Mattei L, Del Bene M, Aiani L, Saini M, Casali C, et al: Intraoperative cerebral glioma characterization with contrast enhanced ultrasound. BioMed Res Int 2014:484261, 2014

25. Prada F, Perin A, Martegani A, Aiani L, Solbiati L, Lamperti $\mathrm{M}$, et al: Intraoperative contrast-enhanced ultrasound for brain tumor surgery. Neurosurgery 74:542-552, 2014

26. Reid MH: Ultrasonic visualization of a cervical cord cystic astrocytoma. AJR Am J Roentgenol 131:907-908, 1978

27. Rygh OM, Selbekk T, Torp SH, Lydersen S, Hernes TA, Unsgaard G: Comparison of navigated 3D ultrasound findings with histopathology in subsequent phases of glioblastoma resection. Acta Neurochir (Wien) 150:10331042, 2008

28. Sanai N, Polley MY, McDermott MW, Parsa AT, Berger MS: An extent of resection threshold for newly diagnosed glioblastomas. J Neurosurg 115:3-8, 2011

29. Selbekk T, Jakola AS, Solheim O, Johansen TF, Lindseth F, Reinertsen I, et al: Ultrasound imaging in neurosurgery: approaches to minimize surgically induced image artefacts for improved resection control. Acta Neurochir (Wien) 155:973-980, 2013

30. Sidhu PS, Choi BI, Nielsen MB: The EFSUMB Guidelines on the non-hepatic clinical applications of contrast enhanced ultrasound (CEUS): a new dawn for the escalating use of this ubiquitous technique. Ultraschall Med 33:5-7, 2012

31. Solheim O, Selbekk T, Jakola AS, Unsgård G: Ultrasoundguided operations in unselected high-grade gliomas-overall results, impact of image quality and patient selection. Acta Neurochir (Wien) 152:1873-1886, 2010

32. Šteňo A, Karlík M, Mendel P, Č́́k M, Šteňo J: Navigated three-dimensional intraoperative ultrasound-guided awake resection of low-grade glioma partially infiltrating optic radiation. Acta Neurochir (Wien) 154:1255-1262, 2012

33. Šteňo A, Matejčík V, Šteňo J: Intraoperative ultrasound in low-grade glioma surgery. Clin Neurol Neurosurg 135:9699, 2015

34. Stummer W, Novotny A, Stepp H, Goetz C, Bise K, Reulen HJ: Fluorescence-guided resection of glioblastoma multiforme by using 5-aminolevulinic acid-induced porphyrins: a prospective study in 52 consecutive patients. $\mathbf{J}$ Neurosurg 93:1003-1013, 2000

35. Stupp R, Hegi ME, Mason WP, van den Bent MJ, Taphoorn MJ, Janzer RC, et al: Effects of radiotherapy with concomitant and adjuvant temozolomide versus radiotherapy 
alone on survival in glioblastoma in a randomised phase III study: 5-year analysis of the EORTC-NCIC trial. Lancet Oncol 10:459-466, 2009

36. Stupp R, Mason WP, van den Bent MJ, Weller M, Fisher $\mathrm{B}$, Taphoorn MJ, et al: Radiotherapy plus concomitant and adjuvant temozolomide for glioblastoma. N Engl J Med 352:987-996, 2005

37. Torzilli G: Contrast-enhanced intraoperative ultrasonography in surgery for liver tumors. Eur J Radiol 51 Suppl:S25-S29, 2004

38. von Herbay A, Westendorff J, Gregor M: Contrast-enhanced ultrasound with SonoVue: differentiation between benign and malignant focal liver lesions in 317 patients. J Clin Ultrasound 38:1-9, 2010

\section{Disclosures}

The authors report no conflict of interest concerning the materi- als or methods used in this study or the findings specified in this paper.

\section{Author Contributions}

Conception and design: Prada, Del Bene. Acquisition of data: Prada, Fornaro, Vetrano, Pollo, DiMeco. Analysis and interpretation of data: Prada, Del Bene, Fornaro, Pollo. Drafting the article: Prada, Del Bene, Mauri. Critically revising the article: Prada, Del Bene, Martegani, Aiani, Sconfienza, Mauri, Solbiati, DiMeco. Reviewed submitted version of manuscript: Prada, Del Bene, Mauri, DiMeco. Approved the final version of the manuscript on behalf of all authors: Prada. Study supervision: DiMeco.

\section{Correspondence}

Francesco Prada, Fondazione IRCCS Istituto Neurologico C. Besta, via Celoria 11, Milan 20133, Italy. email: francesco. prada@istituto-besta.it. 